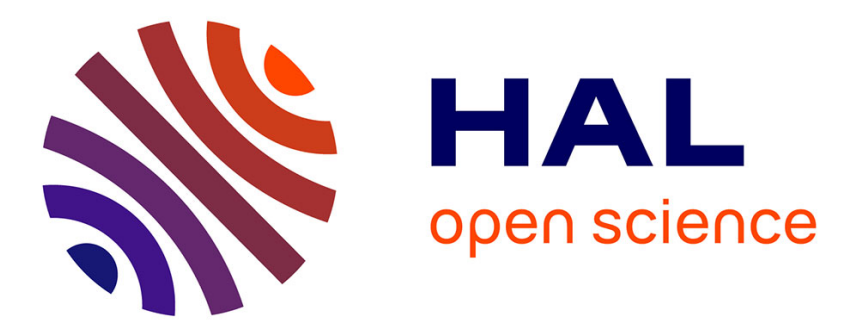

\title{
Aircraft 4D trajectories planning under uncertainties
}

Supatcha Chaimatanan, Daniel Delahaye, Marcel Mongeau

\section{To cite this version:}

Supatcha Chaimatanan, Daniel Delahaye, Marcel Mongeau. Aircraft 4D trajectories planning under uncertainties. SSCI 2015, IEEE Symposium Series on Computational Intelligence, IEEE, Dec 2015, Cape Town, South Africa. pp.ISBN: 978-1-4799-7560-0, 10.1109/SSCI.2015.18 . hal-01271435

\section{HAL Id: hal-01271435 \\ https://hal-enac.archives-ouvertes.fr/hal-01271435}

Submitted on 9 Feb 2016

HAL is a multi-disciplinary open access archive for the deposit and dissemination of scientific research documents, whether they are published or not. The documents may come from teaching and research institutions in France or abroad, or from public or private research centers.
L'archive ouverte pluridisciplinaire HAL, est destinée au dépôt et à la diffusion de documents scientifiques de niveau recherche, publiés ou non, émanant des établissements d'enseignement et de recherche français ou étrangers, des laboratoires publics ou privés. 


\title{
Aircraft 4D trajectories planning under uncertainties
}

\author{
Supatcha Chaimatanan \\ ENAC, MAIAA, \\ F-31055 Toulouse, France \\ Univ de Toulouse, \\ IMT, F-31400 Toulouse, France \\ Email: supatcha@recherche.enac.fr \\ Daniel Delahaye \\ ENAC, MAIAA, \\ F-31055 Toulouse, France \\ Univ de Toulouse, \\ IMT, F-31400 Toulouse, France \\ Email: daniel@recherche.enac.fr \\ Marcel Mongeau \\ ENAC, MAIAA, \\ F-31055 Toulouse, France \\ Univ de Toulouse, \\ IMT, F-31400 Toulouse, France \\ Email: mongeau@recherche.enac.fr
}

February 9, 2016

\begin{abstract}
To sustain the rapidly increasing air traffic demand, the future air traffic management system will rely on a concept, called Trajectory-Based Operations (TBO), that will require aircraft to follow an assigned 4D trajectory (time-constrained trajectory) with high precision. TBO involves separating aircraft via strategic (long-term) trajectory deconfliction rather than the currently-practicing tactical (short-term) conflict resolution. In this context, this paper presents a strategic 4D aircraft trajectory planning approach aiming at minimizing interaction between aircraft trajectories for a given day. The proposed methodology allocates an alternative departure time, a horizontal flight path, and a flight level to each flight at a country and a continent scale. Uncertainties of aircraft position and arrival time on its curvilinear abscissa are taken into account in the
\end{abstract}


trajectory planning process. The proposed approach optimizes the 4D trajectory of each aircraft so as to minimize the interaction between trajectories. A hybrid-metaheuristic optimization algorithm has been developed to solve this large-scale mixed-variable optimization problem. The algorithm is implemented and tested with real air traffic data taking into account uncertainty over the French and the European airspace for which a conflict-free and robust $4 \mathrm{D}$ trajectory plan is produced.

\section{Introduction}

To ensure safety of aircraft traveling from an origin to a destination airport while minimizing delays and congestion, Air Traffic Management (ATM) system manages the air traffic to separate aircraft by some prescribed distance, noted $N_{v}$ for the vertical separation and $N_{h}$ for the horizontal separation. Current ATM regulations require aircraft operating in the terminal maneuvering area (TMA) ${ }^{1}$ to be vertically separated by at least $N_{v}=1,000$ feet (ft) and horizontally separated by a minimum of $N_{h}=3$ nautical miles (NM). In the en-route environment, for aircraft operating up to (and including) $\mathrm{FL}^{2}$ 410, the horizontal minimum separation is increased to $5 \mathrm{NM}$; for aircraft operating above FL 410, the vertical separation is increased to $2,000 \mathrm{ft}$ [1].

Aircraft are considered to be in conflict when these minimum separation requirements are violated. Such conflict situations would not necessary lead to a collision; however, it is a situation that controllers must avoid. One can consider that at any given time, each aircraft has a bounded and closed reserved block of airspace defined by a three-dimensional cylinder, as shown in Figure 1, in which other aircraft are not allowed to enter.

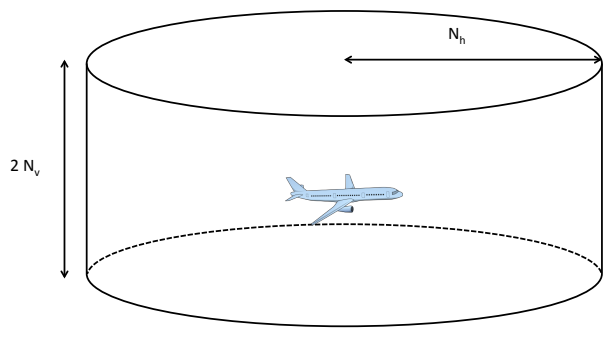

Figure 1: The cylindrical protection volume

Currently, the world's major ATM systems are being modernized in order to accommodate the increasing air traffic demand. The new ATM system will rely on a concept of Trajectory Based Operations (TBOs) which will focus more

\footnotetext{
${ }^{1} \mathrm{~A}$ terminal control area (also known as a terminal maneuvering area) is a controlled airspace surrounding major airports, generally designed as a cylindrical or up-side-down wedding-cake shape airspace of 30 to 50 mile radius and high of 10,000 feet.

${ }^{2}$ Flight level (FL) is a pressure altitude, expressed in hundreds of feet, e.g. and altitude of 32,000 feet is referred to as FL 320 .
} 
on managing each aircraft trajectory so as to adapt the airspace user's demand to the current airspace capacity. In this concept, an aircraft flying through the airspace will be required to follow a negotiated conflict-free trajectory, accurately defined in 4 dimensions ( 3 spatial dimensions and time). This 4D trajectory concept will significantly reduce the need of controller's intervention during the tactical phase. Therefore, a controller will be able to accommodate more flights in a given airspace at a given time.

In this paper, we propose a methodology to address such a strategic 4D trajectory planning at the country and continent scale. The proposed methodology separates aircraft trajectories by allocating alternative route, alternative flight level, and alternative departure time to each flight. Instead of trying to solve each conflict locally, we separate aircraft trajectory in the 3D space and in the time domain so as to minimize global interaction between them. The interaction is an indicator that defines a situation when two or more aircraft trajectories compete for the same space at the same period of time. This route, flight level, and departure time allocation problem is formulated under the form of mixed-integer optimization problem. The objective is to minimize interaction between aircraft trajectories.

As in reality, the aircraft may subject to external events such as wind, temperature, passenger delays, etc. These induce uncertainty on aircraft position and arrival time to a given point. To increase robustness to the $4 \mathrm{D}$ conflictfree trajectory, these uncertainty must be taken into account in the trajectory planning process. In this study, the proposed methodology takes into account uncertainty of aircraft position and in the time domain. A set of alternative 4D trajectories with minimum interaction are obtained through an optimization process that relies on a hybrid-metaheuristic optimization process. To our knowledge, no other research work addresses globally such a 4D strategic planning problem taking into account uncertainty in both 3D space and time domain for such large-scale problems (continental airspace).

The following section of this paper are organized as follows. Section 2 reviews previous related work on strategic trajectory planning problem. Section 3 presents the mathematical model of the 4D trajectory planning problem under uncertainty that we are considering. After that, a method to compute the value of the objective function under such uncertainties is detailed in section 4 . In section 5, a resolution algorithm to solve this problem is discussed. Numerical results from computational experiments are presented in section 6 . Finally, conclusions and perspectives are discussed in section 7 .

\section{Previous related works}

Numerous researches on the ATM problem have been conducted in the past decades. We refer the reader interested by a survey on modeling and optimization in air traffic to the recent book [2]. A survey on mathematical optimization models for air traffic management problems based on different air traffic management strategies is provided in [3]. A comparison of different optimization 
methods (deterministic and metaheuristic optimization approaches) used for ATM is provided in [4].

In the strategic planning (before takeoff) framework, aircraft trajectories can be separated by, for example, modifying the departure time of aircraft such as in [5], [6], and [7]. This method is effective since it absorb the delay on the ground without inducing extra fuel consumption. However, with increasing air traffic demand, significant delays still have to be assigned to a large number of aircraft to meet all airspace-sector and airport capacity constraints.

In [8] and [9], another idea to separate trajectories based on speed regulations is presented. This method is effective at the fine-grain level, however, it is irrelevant in the strategic trajectory planning context. Other strategies consider rerouting, or modifying the flight levels, or a combination of the above-mentioned methods, for example, in [10] and [11]. In [12, 13, 14] the authors show that the departure-time and alternative-route allocation problem is NP hard. However, these works propose improvements of air traffic at the airspace sector level but do not manage conflicts. In [15, 16], the authors focus on minimizing congestion in the airspace sectors by allocating to each flight optimal departure times and alternative routes using genetic algorithms (GA). Their results show that GA is very efficient in solving highly complex problems, however, it is not well adapted for the large-scale 4D trajectory planning problems that we are considering, due to excessive memory requirement intrinsic to population-based optimization algorithms.

The concept of 4D strategic deconfliction that aims to generate conflict-free trajectories for aircraft from origin to destination airports is introduced in the Innovative Future Air Transport System (IFATS) project [17] and the 4 Dimension Contract-Guidance and Control (4D CO-GC) project [18]. In [19, 20], preliminary studies on the optimization of individual $4 \mathrm{D}$ trajectories are presented. In these papers, optimal (conflict-free) 4D trajectories for individual flights are allocated by solving a combinatorial optimization problem using a non-population-based hybrid-metaheuristic optimization method. The numerical results presented in [20] show advantages of the hybrid-metaheuristic optimization approach on ATFM problems. However, the discretization of the search domain (candidate departure times and trajectories) induces high combinatorics.

In [21], the authors extend the works in $[19,20]$ by introducing a method to consider uncertainties of aircraft position in the horizontal plane along the trajectory. The uncertainties are modeled as a disk around the nominal assigned position. Alternative route and departure time for each flight is obtained by solving a mixed-integer optimization problem through a hybrid-metaheuristic optimization algorithm. There exists several conflict detection and resolution methods. We refer the reader interested by a review of this subject to [22].

In this paper, we put forward the work presented in [21]. In addition to the uncertainties in the horizontal plane, we also consider uncertainties of aircraft altitude during climb and descent phase. Moreover, we introduce a method to take into account uncertainty in the time domain. At least to our knowledge, no other research work is able to address robustness of the resulting conflict- 
free $4 \mathrm{D}$ trajectory in presence of uncertainty in the time domain for a large problem (e.g. national and continent size air-traffic). In addition, we describe a method to The interaction to adapted the interaction detection method presented in [21] in order to take into account such uncertainties. We also improve the hybrid-metaheuristics optimization algorithm proposed in [21] by introducing new neighborhood function and new local search steps. Finally, we prove the viability of the overall methodology on large-scale air traffic data on the European continent airspace $(\approx 30,000$ trajectories $)$ including the traffic in the TMA.

\section{Mathematical model}

This section describes the proposed strategic trajectory planning methodology in a mathematical framework. More precisely, the strategic trajectory planning problem under uncertainties that we are considering can be presented as follows:

- We are given a set of flight plans for a given day associated with a nationwide scale or continent-scale air traffic.

- For each flight, $i$, we suppose that the following elements are known:

- a set of possible routes;

- a set of possible flight levels;

- a set of possible departure times;

- the features of the uncertainties of aircraft position and arrival time.

- The goal is to separate the given set of aircraft trajectories in both the three dimensional space and in the time domain by allocating an alternative flight plan (route, departure time, FL) to each flight so as to minimize the global interaction between trajectories.

\subsection{Uncertainty model}

To consider the uncertainty of aircraft position and arrival time, we characterize the uncertainty sets as follows.

\subsubsection{Uncertainty of aircraft position in the horizontal plane}

Consider an initial 4D trajectory planning specifying that an aircraft must arrive at a given horizontal point $(x, y)$ at time $t$. Due to uncertainties, we shall assume that the real horizontal position, $\left(x^{r}, y^{r}\right)$, of the aircraft at time $t$ can be in an area defined by a disk of radius $R_{h}$ (defined by the user) around $(x, y)$, as illustrated in Figure 2. Let $\epsilon_{h_{x}}=\left(x^{r}-x\right)$ and $\epsilon_{h_{y}}=\left(y^{r}-y\right)$ denote the uncertainties of aircraft position in the $x$ and the $y$ directions respectively. 
The vector of uncertainty of aircraft position in the horizontal plane, denoted $\epsilon_{h}=\left\{\epsilon_{h_{x}}, \epsilon_{h_{y}}\right\}$, must belong to the set:

$$
\mathcal{U}_{h}:=\left\{\epsilon_{h}:\left\|\epsilon_{h}\right\|_{2} \leq R_{h}\right\}
$$

In other words, the possible locations of the aircraft at time $t$ are the elements of the set: $\left\{\left(x^{r}, y^{r}\right):\left(x^{r}-x\right)^{2}+\left(y^{r}-y\right)^{2} \leq R_{h}^{2}\right\}$. To ensure horizontal separation of aircraft subjected to such uncertainties, the protection volume has to be enlarged by a radius of $R_{h}$ as illustrated in Figure 2. Thus, the robust minimum separation in the horizontal plane, $N_{h}^{r}$, is defined as:

$$
N_{h}^{r}:=N_{h}+R_{h},
$$

where $N_{h}$ is the (usual) minimum horizontal separation of the case without uncertainty.

\subsubsection{Uncertainty of aircraft position in the vertical dimension}

Aircraft position may be subject to uncertainty in the vertical dimension mainly when the aircraft is not in its cruise phase, e.g. climb, descent.We shall assume that during such a non-level flight phase, the real altitude, denoted $z^{r}$, of the aircraft at a given time $t$ lies in a bounded interval defined by an uncertainty radius $R_{v}$ (set by the user) which reduces strongly when the aircraft reaches its requested flight level. The uncertainty of aircraft position in the vertical dimension, noted $\epsilon_{v}=z^{r}-z$, must therefore belong to the set:

$$
\mathcal{U}_{v}:=\left\{\epsilon_{v}:\left|\epsilon_{v}\right| \leq R_{v}\right\} .
$$

In other words, the possible altitudes of the aircraft during non-level flight phase at time $t$ are the elements of the set: $\left\{z^{r}: z-R_{v} \leq z^{r} \leq z+R_{v}\right\}$. To ensure vertical separation of aircraft subjected to such uncertainties, the vertical separation requirement has to be enlarged by $R_{v}$ as illustrated in Figure 2. Thus, the robust minimum separation in the vertical dimension, noted $N_{v}^{r}$, is defined as:

$$
N_{v}^{r}:=N_{v}+R_{v},
$$

where $N_{v}$ is the (usual) minimum vertical separation of the case without uncertainty.

\subsubsection{Uncertainty of aircraft arrival time}

In addition to the uncertainty in the 3D space domain, aircraft may be subject to uncertainty so that it arrives at a given position with a time error. Let $t_{\epsilon}$ be the maximum time error (defined by the user). For simplicity, to implement the interaction detection scheme, we shall assume that $t_{\epsilon}$ is chosen so that it is a multiple of the discretization time step $t_{s}$. The real arrival time, noted $t^{r}$, of aircraft at the same trajectory point therefore lies in the time interval: 


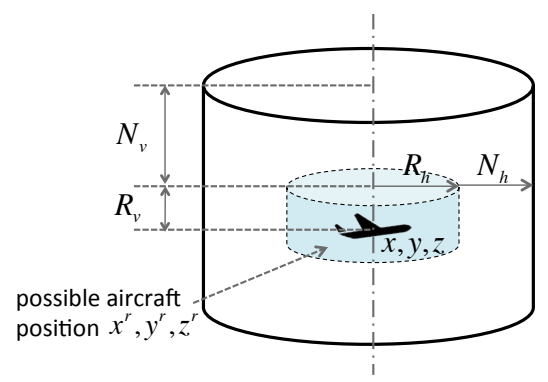

Figure 2: Possible aircraft position in the 3D space domain in presence of deterministic uncertainty.

$\left[t-t_{\epsilon}, t+t_{\epsilon}\right]$. The uncertainty of the arrival time, noted $\epsilon_{t}=t-t^{r}$, must therefore belong to the set:

$$
\mathcal{U}_{t}:=\left\{\epsilon_{t}:\left|\epsilon_{t}\right| \leq t_{\epsilon}\right\} .
$$

We refer the reader interested by the modeling of uncertainties to [23].

\subsection{Interaction between trajectories}

The concept of interaction has been introduced in [21]. To measure the interaction between aircraft trajectories taking into account the above-mentioned deterministic-type uncertainties, let us first consider two trajectories A and B illustrated in Figure 3, and let $P$ and $Q$ be any pair of sample points on the trajectories A and B respectively. Then, we must check whether the minimum separations, $N_{h}^{r}$ and $N_{v}^{r}$ is satisfied, between every possible pair of points such as $P$ and $Q$ (pair-wise comparisons). Let $t_{P}^{r}$ be the real arrival time of aircraft

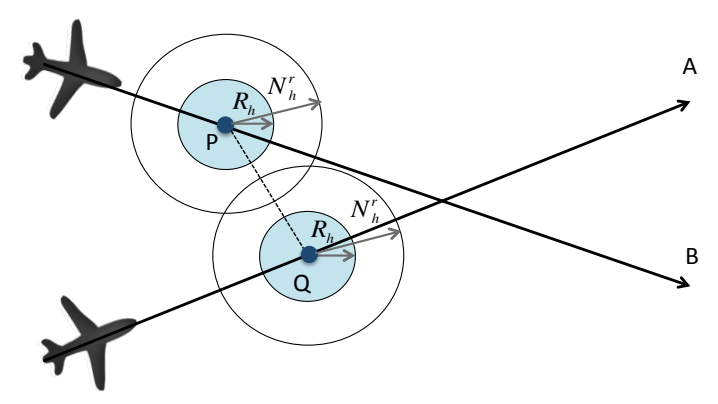

Figure 3: Evaluating the interaction between two continuous trajectories A and $\mathrm{B}$ in presence of deterministic-type uncertainty.

A at point $P$, and let $t_{Q}^{r}$ be the real arrival time at point $Q$, therefore:

$$
t_{P}^{r} \in\left[t_{P}-t_{\epsilon}, t_{P}+t_{\epsilon}\right]
$$


and

$$
t_{Q}^{r} \in\left[t_{Q}-t_{\epsilon}, t_{Q}+t_{\epsilon}\right]
$$

respectively.

A potential conflict between trajectories $\mathrm{A}$ and $\mathrm{B}$, taking into account uncertainties, can occur when the three following conditions are satisfied for a certain pair of sample points, $P$ and $Q$, from each trajectory:

- $d_{h}:=\sqrt{\left(x_{P}-x_{Q}\right)^{2}+\left(y_{P}-y_{Q}\right)^{2}}<N_{h}^{r}$.

- $d_{v}:=\left|z_{P}-z_{Q}\right|<N_{v}^{r}$.

- $\left[t_{P}-t_{\epsilon}, t_{P}+t_{\epsilon}\right] \cap\left[t_{Q}-t_{\epsilon}, t_{Q}+t_{\epsilon}\right] \neq \emptyset$, i.e. $\left|t_{P}-t_{Q}\right| \leq 2 t_{\epsilon}$.

When the above conditions are satisfied, we say that point $P$ is in conflict with point $Q$ taking into account the deterministic-type uncertainty.

Figure 4 illustrates the four possible scenarios of arrival time of two aircraft to the same 3D space region. Remark that a potential conflict between $P$ and $Q$ can occur only in cases a) and case b) where $\left|t_{P}-t_{Q}\right| \leq 2 t_{\epsilon}$.

Let us define further

$$
\mathcal{C}^{D}(P, Q)= \begin{cases}1 & \text { if point } P \text { is in conflict with point } Q \\ & \text { taking into account the uncertainty } \\ 0 & \text { otherwise. }\end{cases}
$$

We defined the interaction, denoted $\Phi_{i, k}^{D}(u)$, at point $P_{i, k}\left(u_{i}\right)$ to be the sum of all the conflicts associated to point $P_{i, k}\left(u_{i}\right)$ :

$$
\Phi_{i, k}^{D}(u):=\sum_{\substack{j=1 \\ j \neq i}}^{N} \sum_{l=1}^{K_{j}} \mathcal{C}^{D}\left(P_{i, k}\left(u_{i}\right), P_{j, l}\left(u_{j}\right)\right),
$$

where $K_{j}$ is the number of sampling points for trajectory $j$.

Let us now denote $\epsilon$ to be the uncertainty of aircraft positions and aircraft arrival times, and let $\mathcal{U}=\mathcal{U}_{h} \times \mathcal{U}_{v} \times \mathcal{U}_{t}$ be the uncertainty set, where $\mathcal{U}_{h}, \mathcal{U}_{v}$, and $\mathcal{U}_{t}$ are defined by (1), (2), and (3) respectively. The robust interaction associated to the point $P_{i, k}\left(u_{i}\right)$ considering the deterministic-type uncertainty, denoted $\Phi_{i, k}^{D}(u)$, can be defined as:

$$
\Phi_{i, k}^{D}(u)=\sup _{\epsilon \in \mathcal{U}} \Phi_{i, k}(u, \epsilon)
$$

With the above definitions, we can perform implicitly the supremum computation involved in equation (5). Indeed, one can straightforwardly check that we have

$$
\Phi_{i, k}^{D}(u)=\sum_{\substack{j=1 \\ j \neq i}}^{N} \sum_{l=1}^{K_{j}} \mathcal{C}^{D}\left(P_{i, k}\left(u_{i}\right), P_{j, l}\left(u_{j}\right)\right)
$$




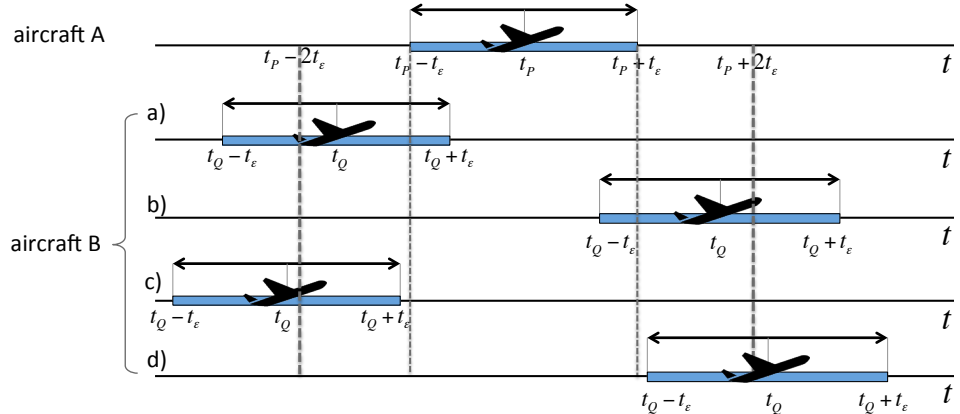

Figure 4: Possible scenarios of arrival time of two aircraft to the same 3D space region.

\subsection{Trajectory separation maneuvers}

In this section, we describe three possible trajectory separation maneuvers, that we are considering in order to generate alternative $4 \mathrm{D}$ trajectories that minimize the total interactions.

\subsubsection{Alternative departure time}

The departure time of each flight, $i$, can be shifted by a positive (delay) or a negative (advance) time shift denoted by $\delta_{i}$. The departure time, $t_{i}$, of flight $i$ is therefore $t_{i}=t_{i, 0}+\delta_{i}$, where $t_{i, 0}$ is the initially-planned departure time of flight $i$. Following common practice in airports, the set of possible values for $\delta_{i}$ will be discrete.

\subsubsection{Alternative trajectory design}

A method to generate an alternative trajectory that we consider in the paper is described in [21]. To generate an alternative route, we modify the (given) initial horizontal flight profile, using a set of virtual waypoints.

As detailed in [21], we define for each flight $i$, a vector, $w_{i}$, of virtual waypoints (our optimization variables) used to control the trajectory shape of flight $i$ :

$$
w_{i}=\left(w_{i}^{1}, w_{i}^{2}, \ldots, w_{i}^{M}\right),
$$

where $M$ denotes the number of virtual waypoints that the user is allowed to introduce, where $w_{i}^{m}=\left(w_{i x^{\prime}}^{m}, w_{i y^{\prime}}^{m}\right)$ is the $m^{t h}$ virtual waypoint of trajectory $i$, and where $w_{i x^{\prime}}^{m}$ and $w_{i y^{\prime}}^{m}$ are the normalized longitudinal and lateral components of $w_{i}^{m}$ respectively. Figure 5, illustrates a possible alternative horizontal profile for a given trajectory constructed with $M=2$ virtual waypoints. 


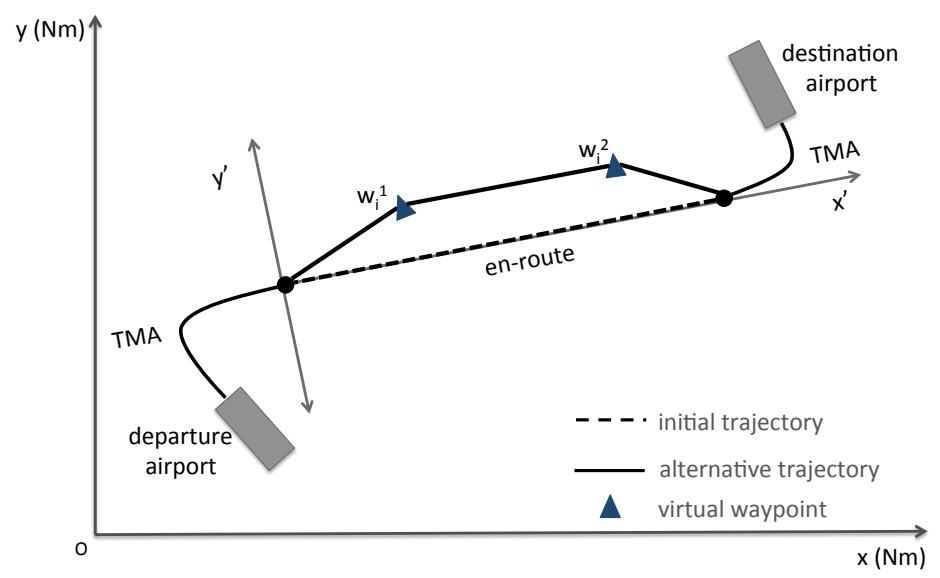

Figure 5: An alternative horizontal profile for a given trajectory, $i$, constructed with $M=2$ virtual waypoints.

\subsubsection{Alternative flight level}

We define another decision variable associated to each flight $i$ : a flight-level shift $l_{i}$. Therefore, the flight level, $F L_{i}$, of flight $i$ is given by:

$$
F L_{i}=F L_{i, 0}+l_{i},
$$

where $F L_{i, 0}$ is the (given data) initially-planned flight level of flight $i$. Figure 6 shows a trajectory with two alternative flight levels.

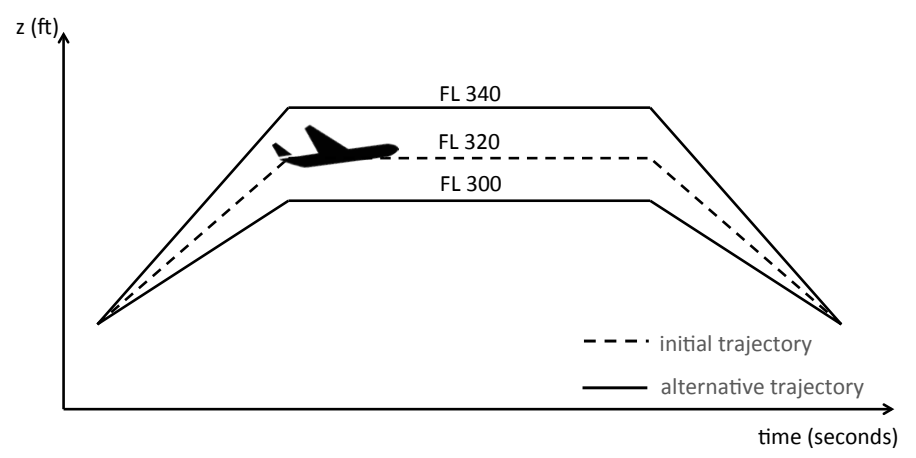

Figure 6: Two alternative vertical profiles for a trajectory (two alternative flight levels). 


\subsection{Mixed-integer programming formulation}

\subsubsection{Decision variables.}

Let us set the compact vector notation:

$$
\begin{aligned}
\boldsymbol{\delta} & :=\left(\delta_{1}, \delta_{2}, \ldots, \delta_{N}\right), \\
\mathbf{w} & :=\left(w_{1}, w_{2}, \ldots, w_{N}\right),
\end{aligned}
$$

and

$$
\mathbf{l}:=\left(l_{1}, l_{2}, \ldots, l_{N}\right)
$$

We shall denote by $u_{i}$ the components of $u$. It is a vector whose components are related to the modification of the $i^{\text {th }}$ trajectory, thereby our decision variable is:

$$
u:=(\boldsymbol{\delta}, \mathbf{l}, \mathbf{w})
$$

\subsubsection{Constraints}

To minimize interaction between trajectories, we consider the following constraints:

Allowed departure time shift. Since it is not reasonable to delay or to advance departure times for too long, the departure time shift, $\delta_{i}$, is assumed to be limited to lie in the interval

$$
\left[\delta_{a}^{i}, \delta_{d}^{i}\right]
$$

Common practice in airports conducted us to rely on a discretization of this time interval. Given the (user-defined) time-shift step size $\delta_{s}$, this yields $N_{a}^{i}:=\frac{-\delta_{a}^{i}}{\delta_{s}}$ possible advance slots and $N_{d}^{i}:=\frac{\delta_{d}^{i}}{\delta_{s}}$ possible delay slots of flight $i$. Therefore, we define the set, $\Delta_{i}$, of all possible departure time shifts of flight $i$ by

$$
\begin{aligned}
\Delta_{i}:= & \left\{-N_{a}^{i} . \delta_{s},-\left(N_{a}^{i}-1\right) \cdot \delta_{s}, \ldots,\right. \\
& \left.-\delta_{s}, 0, \delta_{s}, \ldots,\left(N_{d}^{i}-1\right) . \delta_{s}, N_{d}^{i} \cdot \delta_{s}\right\} .
\end{aligned}
$$

Maximum allowed flight-level changes. To limit the change of flight levels, the flight level shift is also bounded. The set, $\Delta F L_{i}$, of all possible flight-level shifts for flight $i$ is:

$$
\begin{aligned}
\Delta F L_{i}:= & {\left[F L_{i, 0}-l_{i, \max }, \ldots, F L_{i, 0}\right.} \\
& \left.-1,0, F L_{i, 0}+1, \ldots, F L_{i, 0}+l_{i, \max }\right],
\end{aligned}
$$

where $l_{i, \max }$ is the (user-provided) maximum flight level shifts allowed to be allocated to flight $i$.

Maximal route length extension. The maximal route length extension should be limited so that it is acceptable by the airline. Let $0 \leq d_{i} \leq 1$ be the maximum allowed route length extension coefficient of flight $i$ (model parameter 
to be set by the user). To restrain the route length extension, the alternative en-route profile of flight $i$ must satisfy:

$$
L_{i}\left(w_{i}\right) \leq\left(1+d_{i}\right),
$$

where $L_{i}\left(w_{i}\right)$ denotes the normalized length of the alternative en-route profile determined by $w_{i}$. The length $L_{i}\left(w_{i}\right)$ can be straightforwardly computed once the position of the waypoints (contained in the vector $w_{i}$ ) is known. Constraint can in fact be implicitly satisfied by restricting the set of possible waypoint locations (as will be described below).

Allowed waypoint locations. As detailed in [21, 23], to limit the search space, to prevent undesirable sharp turns, and to restrain the route length extension, we bound the possible location of each virtual waypoint. To avoid sharp turns, the longitudinal position of the virtual waypoints should not be too close to each other.

Let $W_{i x^{\prime}}^{m}$ be a set of all possible normalized longitudinal locations of the $m^{\text {th }}$ virtual waypoint on trajectory $i$. For each trajectory $i$, the normalized longitudinal component, $w_{i x^{\prime}}^{m}$, is set to lie in the interval:

$$
W_{i x^{\prime}}^{m}:=\left[\left(\frac{m}{1+M}-b_{i}\right),\left(\frac{m}{1+M}+b_{i}\right)\right] .
$$

To obtain a regular trajectory, the normalized longitudinal component of two adjacent waypoints must not overlap, i.e.

$$
\left(\frac{m}{1+M}+b_{i}\right)<\left(\frac{m+1}{1+M}-b_{i}\right)
$$

and hence the user should choose $b_{i}$ so that

$$
b_{i}<\frac{1}{2(M+1)} .
$$

Let $W_{i y^{\prime}}^{m}$ be a set of all possible normalized lateral locations of the $m^{\text {th }}$ virtual waypoint on trajectory $i$. Similarly, the normalized lateral component, $w_{i y^{\prime}}^{m}$, is restricted to lie in the interval:

$$
W_{i y^{\prime}}^{m}:=\left[-a_{i}, a_{i}\right]
$$

where $0 \leq a_{i} \leq 1$ is a (user-defined) model parameter chosen a priori so as to satisfy (9).

\subsubsection{Objective function.}

The objective is to minimize the total interaction between trajectories taking into account uncertainties. Therefore, the robust total interaction between trajectories, that we are minimizing, is:

$$
\Phi_{t o t}^{D}(u)=\sum_{i=1}^{N} \sum_{k=1}^{K_{i}} \Phi_{i}^{D}(u),
$$


where $N$ is the total number of trajectories.

To summarize, the strategic trajectory planning problem, based on deterministictype uncertainty set, can be represented by an interaction minimization problem formulated as a mixed-integer optimization problem as follows:

$$
\min _{u} \Phi_{t o t}^{D}(u)
$$

subject to

$$
\begin{aligned}
& \delta_{i} \in \Delta_{i}, \quad i=1,2, \ldots, N \\
& l_{i} \in \Delta F L_{i}, \quad i=1,2, \ldots, N \\
& w_{i}^{m} \in W_{i x^{\prime}}^{m} \times W_{i y^{\prime}}^{m}, \quad m=1,2, \ldots, M, \\
& \quad i=1,2, \ldots, N,
\end{aligned}
$$

where $\Phi_{\text {tot }}^{D}(u)$ is defined by $(14)$, and $\Delta_{i}, \Delta F L_{i}, W_{i x^{\prime}}^{m}$, and $W_{i y^{\prime}}^{m}$ are defined by (7), (8), (10), and (13) respectively.

\section{Interaction detection method}

The interaction between trajectories is measured using the method proposed in $[21,23]$. To consider this deterministic-type uncertainty, one simply has to adjust the size of the (3D space) grid cells according to the (user-provided) robust minimum separation $N_{h}^{r}$ and $N_{v}^{r}$ (robust grid). Then, to detect interactions, for each cell $\left(I_{x}, I_{y}, I_{z}, I_{t}\right)$ corresponding to each sampling point $P_{i, k}:=\left(x_{P_{i, k}}, y_{P_{i, k}}, z_{P_{i, k}}, t_{P_{i, k}}\right)$, one simply needs to check all the surrounding cells (in the robust grid) corresponding to the time period $\left[t_{P}-2 t_{\epsilon}, t_{P}+2 t_{\epsilon}\right]$. The algorithm used to compute the total interaction between $N$ trajectories taking into account the deterministic-type uncertainty is described in detail in Algorithm 1.

\section{Resolution algorithm}

To solve the robust strategic $4 \mathrm{D}$ trajectory planning problem, we rely on the hybrid Simulated Annealing / Iterative Improvement Local Search (SA / IILS) algorithm proposed in [21]. As we have introduced an additional decision variable, $l_{i}$, to modify the flight level of any given trajectory $i$, some modifications to the neighborhood function are made as follows.

Consider a chosen flight $i$ to be modified, we introduce here another userdefined parameter, noted $P_{l}$, to control the probability to modify the flight level of flight $i$. This parameter $P_{l}$ must satisfy:

$$
P_{w}+P_{l} \leq 1,
$$

where $P_{w}$ is the previously-defined (user-provided) probability to modify the location of waypoints. Finally, the probability to modify the departure time of 


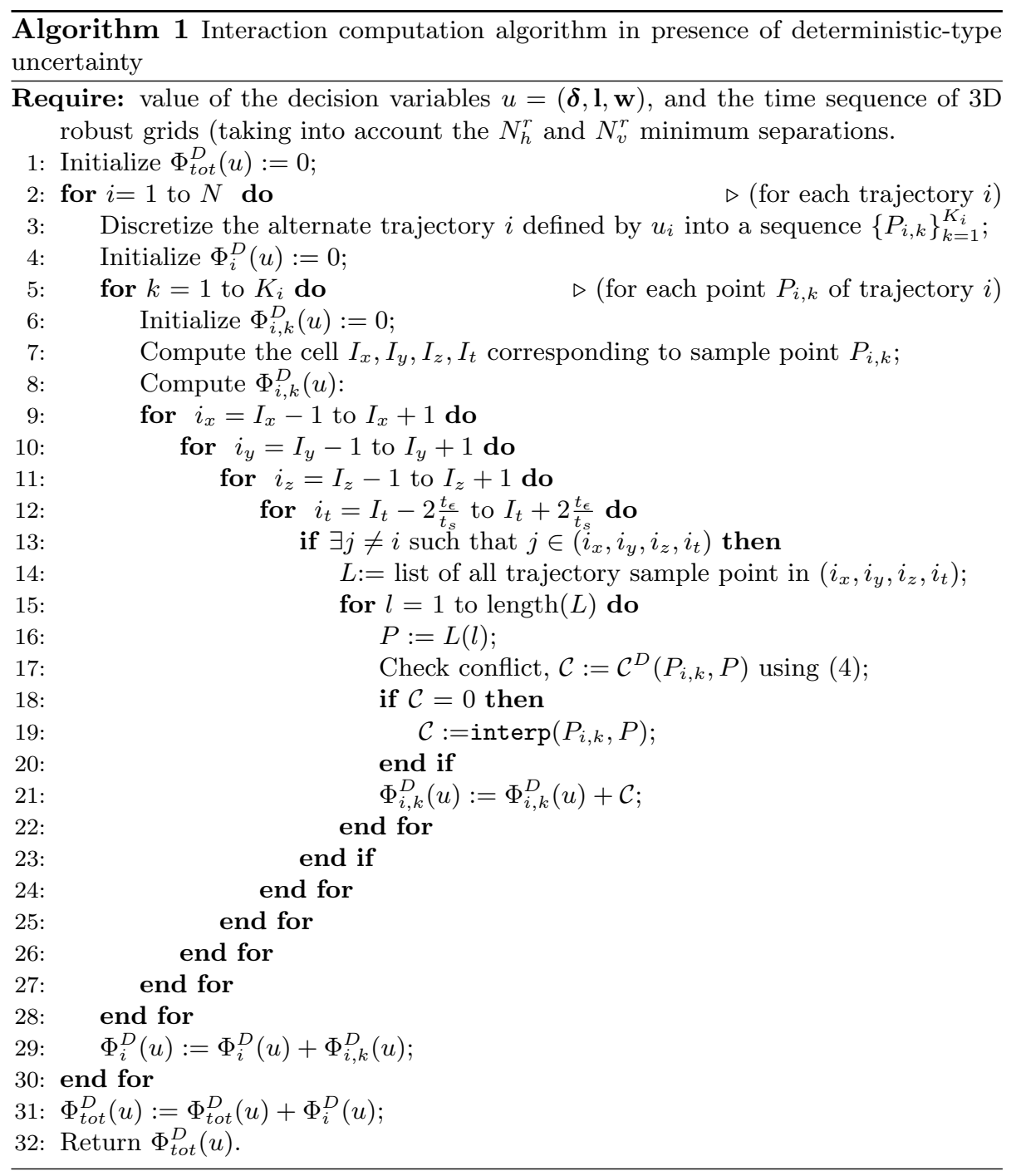




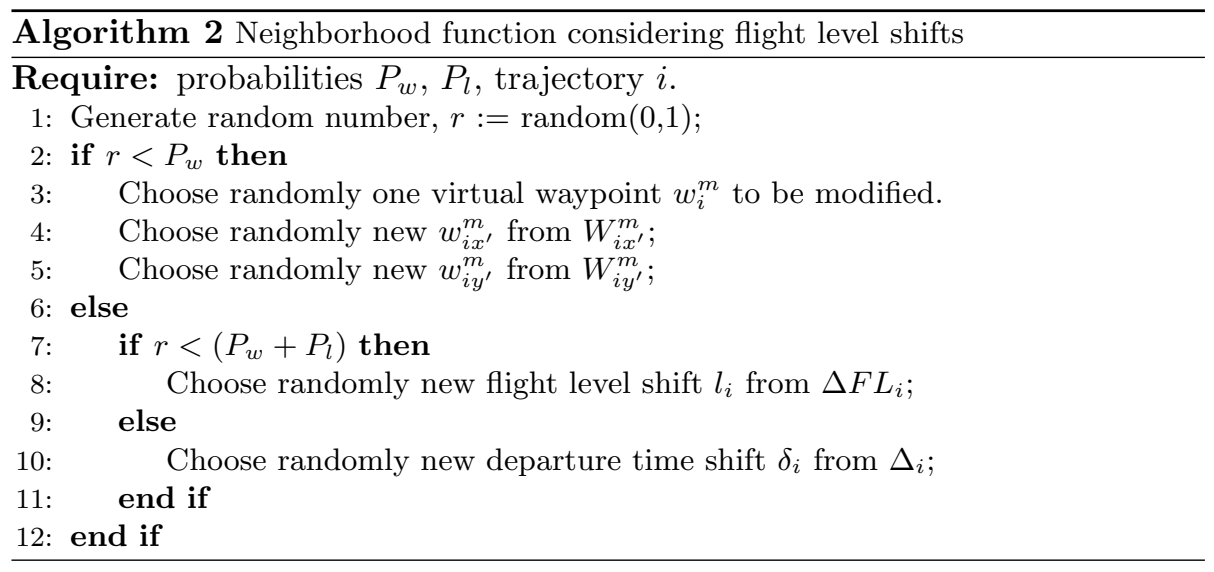

flight $i$ is $1-\left(P_{w}+P_{l}\right)$. The new neighborhood function considering flight level shifts is summarized in Algorithm 2.

Two additional local search algorithms to intensify the search on each particular trajectory in different solution spaces are introduced as follows:

Intensify the search in the Time Domain (TD). This local search module intensifies the search by modifying only the departure time of a given trajectory $i$. The algorithm repeats until a pre-defined number of local-search iterations is performed.

Intensify the search in the Flight-level Domain (FD). This local search module intensifies the search by modifying the flight level of a given trajectory $i$. If the change of flight level yields an improvement of the objectivefunction value, the module further intensifies the search on the current flight level by applying a local change from the neighborhood structure to trajectory $i$ (using the PT local search of [21]). The algorithm repeats until a pre-defined number of local search iterations is performed.

\section{Numerical results}

The strategic $4 \mathrm{D}$ trajectory planning methodology addressing the deterministic type uncertainty is implemented on an AMD Opteron $2 \mathrm{GHz}$ processor with 128 Gb RAM. It is tested with the national-size and continent-size air traffic over the French and the European airspace.

\subsubsection{National-size en-route air traffic}

First, we test the proposed methodology on the full-day national-size en-route air traffic over the French airspace, involving 8,836 trajectories. Simulations are performed with different values for the parameters $R_{h}, R_{v}$, and $t_{\epsilon}$, defining the size of the uncertainty sets.

The parameter values chosen to specify the optimization problem are given 
Table 1: Chosen (user-defined) parameter values specifying the robust optimization problem for the national-size air traffic.

\begin{tabular}{ll}
\hline parameter & value \\
\hline$t_{s}$ & 20 seconds \\
$\delta_{s}$ & 20 seconds \\
$-\delta_{a}^{i}=\delta_{d}^{i}:=\delta$ & 120 minutes \\
$d_{i}$ & 0.20 \\
$l_{i, \max }:=l_{\max }$ & 2 \\
$M$ & 3 \\
\hline
\end{tabular}

Table 2: Empirically-set (user-defined) parameter values of the resolution methodology to solve the national-size air traffic.

\begin{tabular}{ll}
\hline parameter & value \\
\hline Number of iterations at each temperature step, $N_{I}$ & 200 \\
Initial rate of accepting degrading solutions, $\tau_{0}$ & 0.3 \\
Geometrical temperature reduction coefficient, $\beta$ & 0.99 \\
Final temperature, $T_{f}$ & $(1 / 500) . T_{0}$ \\
Inner-loop interpolation sampling time step, $t_{\text {inter }}$ & 5 seconds \\
Probability to modify horizontal flight profile, $P_{w}$ & $1 / 3$ \\
Probability to modify flight level, $P_{l}$ & $1 / 3$ \\
Threshold value, $\Phi_{\tau}$ & $0.5 \Phi_{\text {avg }}$ \\
\hline
\end{tabular}

in Table 1. The parameter values specifying the resolution algorithm are empirically set and given in Table 2. The initial and final total interaction between trajectories, the computation time, and the number of iterations performed to solve the problems considering different levels of uncertainty are reported in Table 3 (the vertical uncertainty radius, $R_{v}$, is used only when aircraft are climbing and descending).

The size of the uncertainty set affects the resolution time and the final total interaction between trajectories. When uncertainties are not considered (case 1), the algorithm reaches interaction-free solution in short computation time. When increasing the time uncertainty, the initial interaction increases significantly (cases 2, 4, 5 and 6), and the algorithm requires more computation time to converge. The algorithm reaches an interaction-free solution for the case 3 . It solves up to $99.7 \%$ of the initial interactions in the remaining cases $(2,4,5$, and 6 ), within computation times that are still compatible in a strategic planning context. 
Table 3: Initial and final total interaction between trajectories for the nationalsize air traffic, considering different dimensions for the uncertainty set (the vertical uncertainty radius, $R_{v}$, is relevant only when aircraft are climbing or descending).

\begin{tabular}{|c|c|c|c|c|c|}
\hline case & $\begin{array}{l}\text { uncertainty set } \\
\text { dimensions }\end{array}$ & $\begin{array}{c}\text { initial } \\
\Phi_{t o t}^{D}\end{array}$ & $\begin{array}{c}\text { solved } \\
\text { interactions }\end{array}$ & $\begin{array}{c}\text { CPU } \\
\text { time (minutes) }\end{array}$ & $\begin{array}{l}\text { no. of } \\
\text { iterations }\end{array}$ \\
\hline 1 & $\begin{array}{l}R_{h}=0 \mathrm{Nm} \\
R_{v}=0 \text { feet. } \\
t_{\epsilon}=0 \mathrm{~s}\end{array}$ & 83,044 & $100.0 \%$ & 18.3 & 18,259 \\
\hline 2 & $\begin{array}{l}R_{h}=0 \mathrm{Nm} \\
R_{v}=0 \text { feet. } \\
t_{\epsilon}=180 \text { seconds. }\end{array}$ & $2,282,436$ & $99.7 \%$ & $1,093.8$ & $1,083,215$ \\
\hline 3 & $\begin{array}{l}R_{h}=1 \mathrm{Nm} \\
R_{v}=100 \text { feet. } \\
t_{\epsilon}=60 \text { seconds. }\end{array}$ & 765,448 & $100.0 \%$ & 101.1 & 97,400 \\
\hline 4 & $\begin{array}{l}R_{h}=1 \mathrm{Nm} . \\
R_{v}=100 \text { feet. } \\
t_{\epsilon}=120 \text { seconds. }\end{array}$ & $1,425,384$ & $99.7 \%$ & $1,809.0$ & $1,791,000$ \\
\hline 5 & $\begin{array}{l}R_{h}=1 \mathrm{Nm} \\
R_{v}=100 \text { feet. } \\
t_{\epsilon}=240 \text { seconds. }\end{array}$ & $2,821,706$ & $98.7 \%$ & $2,213.3$ & $2,191,970$ \\
\hline 6 & $\begin{array}{l}R_{h}=2 \mathrm{Nm} \\
R_{v}=100 \text { feet. } \\
t_{\epsilon}=240 \text { seconds. }\end{array}$ & $5,000,430$ & $97.9 \%$ & $2,289.8$ & $2,266,956$ \\
\hline
\end{tabular}

\subsubsection{Continent-size air traffic}

To test the proposed methodology on continent-size air traffic, simulations were performed on the en-route traffic scenario as well as on traffic involving the TMAs. The data set is a full day of air-traffic over the European airspace on July 1, 2011. It consists of 30,695 trajectories simulated with optimal vertical profiles and with direct route However, due to the lack of data, alternative flight levels for these two continent-size instances are not available. To solve these problem instances, we limit the maximum flight level change, $l_{i, \max }$, to zero, for all flight $i\left(l_{\max }=0\right)$. The uncertainty of aircraft position in the TMA is not taken into account $\left(R_{h}^{T M A}=0\right.$, and $\left.R_{v}^{T M A}=0\right)$, since during this phase of flight, aircraft are usually required to follow a given path with very high precision. The user-defined input parameters of the optimization algorithm are all set to the same values as those for the national-size en-route air traffic (Table 1 ), except for $l_{\max }$ which is set to zero as explained above.

The user-defined parameter values specifying the hybrid SA / IILS algorithm to solve these problem instances are given in Table 2 . The only difference with 
Table 4: Initial and final total interaction between trajectories for the continentscale air traffic with different dimensions for the uncertainty set.

\begin{tabular}{|c|c|c|c|c|c|c|}
\hline case & $\begin{array}{c}\text { traffic } \\
\text { scenario }\end{array}$ & $\begin{array}{l}\text { uncertainty } \\
\text { set dimensions }\end{array}$ & $\begin{array}{c}\text { initial } \\
\Phi_{\text {tot }}^{D}\end{array}$ & $\begin{array}{c}\text { solved } \\
\text { interactions }\end{array}$ & $\begin{array}{l}\text { CPU time } \\
\text { (minutes) }\end{array}$ & $\begin{array}{c}\text { no. of } \\
\text { iterations }\end{array}$ \\
\hline 1 & $\begin{array}{l}\text { only } \\
\text { en-route }\end{array}$ & $\begin{array}{l}R_{h}=0 \mathrm{Nm} \\
R_{v}=0 \text { feet. } \\
t_{\epsilon}=0 \mathrm{~s}\end{array}$ & 142,144 & $100.0 \%$ & 43.1 & 49,000 \\
\hline 2 & $\begin{array}{c}\text { only } \\
\text { en-route }\end{array}$ & $\begin{array}{l}R_{h}=3 \mathrm{Nm} . \\
R_{v}=200 \text { feet. } \\
t_{\epsilon}=60 \mathrm{~s}\end{array}$ & $5,142,632$ & $87.7 \%$ & $2,756.2$ & $2,728,776$ \\
\hline 3 & $\begin{array}{c}\text { only } \\
\text { en-route }\end{array}$ & $\begin{array}{l}R_{h}=3 \mathrm{Nm} . \\
R_{v}=200 \text { feet. } \\
t_{\epsilon}=0 \mathrm{~s} .\end{array}$ & 430,234 & $100.0 \%$ & 347.6 & 345,528 \\
\hline 4 & with TMA & $\begin{array}{l}R_{h}=0 \mathrm{Nm} . \\
R_{v}=0 \text { feet. } \\
t_{\epsilon}=0 \mathrm{~s}\end{array}$ & 235,632 & $100.0 \%$ & 478.1 & 473,345 \\
\hline 5 & with TMA & $\begin{array}{l}R_{h}=0 \mathrm{Nm} . \\
R_{v}=0 \text { feet. } \\
t_{\epsilon}=120 \mathrm{~s}\end{array}$ & $3,874,402$ & $85.5 \%$ & $2,652.1$ & $2,625,714$ \\
\hline 6 & with TMA & $\begin{array}{l}R_{h}=3 \mathrm{Nm} . \\
R_{v}=200 \text { feet. } \\
t_{\epsilon}=0 \mathrm{~s}\end{array}$ & 487,698 & $100.0 \%$ & 578.4 & 572,648 \\
\hline
\end{tabular}

those for the national-size instance (Table 2) is that the number of iterations at each temperature step is increased from 200 to $4,000, P_{l}$ is set to zero, and $P_{w}$ is consequently increased from $1 / 3$ to $1 / 2$.

The initial and final total interaction between trajectories, and the computation time to solve the problem considering different levels of uncertainty are reported in Table 4. Although the trajectories can be separated only by modifying the horizontal flight profile and the departure time of each flight, the resolution algorithm finds an interaction-free solution, taking into account uncertainty of aircraft positions, for both problem instances in cases 3 and 6 . When time uncertainty is considered (case 2 and 5), there remains less than $15 \%$ of the initial interaction between trajectories. This could be improved by introducing more degrees of freedom to the solution space, e.g. alternative flight levels, or speed regulation in the TMA. The most remaining interactions are located in the TMA. In compare with the methodology proposed in [21], the methodology proposed in this paper yields solution that is more robust to uncertainties in the vertical and in time domain. 


\section{Conclusions}

In this paper we have presented a methodology to consider uncertainty of aircraft position and arrival time in the strategic $4 \mathrm{D}$ trajectory planning process. The uncertainties have been modeled with deterministic sets. The algorithm therefore tries to minimize the interaction between trajectories considering all possible scenario implicitly described by the uncertainty sets (worst-case approach). The algorithm developed for the case without uncertainty was adopted via a modification of the way the objective function is evaluated. The modified algorithm was tested on national-size and continent-size air traffic. It was able to find interaction-free solutions for some uncertainty set sizes. There remains less than $15 \%$ of the initial interactions when the size of the uncertainty set is larger.

The level of uncertainty to be considered is a trade-off between the desired robustness of the solution obtained and the associated trajectory modifications costs, to be decided by the user. Considering too important uncertainty in strategic planning will, indeed, results in a lost of capacity, since large portions of airspace have to be cleared for a given aircraft for a long period of time. Instead, the user can consider lower uncertainty levels, and iteratively solve the remaining interactions during pre-tactical and tactical phases.

When the traffic in the TMA is included, result may be improved by adding speed control variable in the state space to reduce interaction in such area which is not the case in the above-presented algorithm (in all benchmark with TMA, the remaining interaction are mainly located in such area).

\section{Acknowledgment}

This work has been supported by 4 Dimension Contract Guidance and Control (4D CO-GC) project, and by the French National Research Agency (ANR) through JCJC program (project ATOMIC n ANR 12-JS02-009-01).

\section{References}

[1] M. Nolan, Fundamentals of Air Traffic Control, 5th ed. Cengage learning, 2011.

[2] D. Delahaye and S. Puechmorel, Modeling and Optimization of Air Traffic. Wiley-ISTE, 2013.

[3] A. Agustín, A. Alonso-Ayuso, L. Escudero, and C. Pizarro, "Mathematical optimization models for air traffic flow management: A review," in A. Bui, I. Tseveendorkj (Eds.), Combinatorial Optimization in Practice. Studia Informatica Universalis: Hermann Informatique, 2010, vol. 8, pp. 141-184. 
[4] J. Lohn and J. Rios, "A comparison of optimization approaches for nationwide traffic flow management," in AIAA Guidance, Navigation and Control Conference, Chicago (Illinois), 2009.

[5] N. Barnier and C. Allignol, "4D - trajectory deconfliction through departure time adjustment," in ATM 2009, the 8th USA/Europe Air Traffic Management Research and Development Seminar, Napa (California), 2009.

[6] A. R. Odoni, "The flow management problem in air traffic control," in Odoni, A.R., Bianco, L., Szego, G. (eds.), Flow Control of Congested Networks. Springer, 1987, pp. 269-288.

[7] M. Terrab and A. R. Odoni, "Strategic flow management for air traffic control," Operations Research, vol. 41, no. 1, pp. 138-152, 1993. [Online]. Available: http://www.jstor.org/stale/171949

[8] S. Cafieri and N. Durand, "Aircraft deconfliction with speed regulation: new models from mixed-integer optimization," Journal of Global Optimization, vol. 58, no. 4, pp. 613-629, April 2014. [Online]. Available: http://link.springer.com/article/10.1007 $\ \% 2 F s 10898-013-0070-1$

[9] S. Constans, B. Fontaine, and R. Fondacci, "Minimizing potential conflict quantity with speed control," in The 4th Eurocontrol Innovative Research Workshop And Exhibition, Bretigny-sur-Orge (France), 2005, pp. 265-274.

[10] D. Gianazza and N. Durand, "Separating air traffic flows by allocating 3D-trajectories," in DASC 2004, the 23rd IEEE/AIAA Digital Avionics Systems Conference, vol. 1, 2004, pp. 2.D.4-21-13 Vol.1.

[11] N. Barnier and C. Allignol, "Combining flight level allocation with ground holding to optimize 4D-deconfliction," in ATM 2011, the 9th USA/Europe Air Traffic Management Research and Development Seminar, Berlin (Germany), 2011.

[12] D. Bertsimas and S. Patterson, "The air traffic flow management problem with en-route capacities," Operations Research, vol. 46, no. 3, pp. 406-422, 1998.

[13] D. Bertsimas, G. Lulli, and A. Odoni, "The air traffic flow management problem: An integer optimization approach," in Integer Programming and Combinatorial Optimization, ser. Lecture Notes in Computer Science. Springer, 2008, vol. 5035, pp. 34-46. [Online]. Available: http://dx.doi.org/10.1007/978-3-540-68891-4_3

[14] — , "An integer optimization approach to large-scale air traffic flow management," Operations Research, vol. 59, no. 2, pp. 211-227, 2011.

[15] S. Oussedik and D. Delahaye, "Reduction of air traffic congestion by genetic algorithms," in Parallel Problem Solving from Nature - PPSN V, ser. Lecture Notes in Computer Science. Springer, 1998, vol. 1498, pp. 855-864. [Online]. Available: http://dx.doi.org/10.1007/BFb0056927 
[16] S. Oussedik, "Application de l'evolution artificielle aux problèmes de congestion du trafic aérien," Ph.D. dissertation, École Polytechnique, Palaiseau (France), 2000.

[17] H. H. Toebben, C. L. Tallec, A. Joulia, J. Speidel, and C. Edinger, "Innovative future air transport system: Simulation of a fully automated ATS," in 26th International Congress of the Aeronautical Sciences, Anchorage, Alaska, 2008.

[18] A. Joulia and C. L. Tallec, "Aircraft 4D contract based operation: The 4DCO-GC project," in 28th International Congress of the Aeronautical Sciences, Brisbane, Australia, 2012. [Online]. Available: http://www.4dcogc-project.org

[19] S. Chaimatanan, D. Delahaye, and M. Mongeau, "A methodology for strategic planning of aircraft trajectories using simulated annealing," in ISIATM 2012, the 1st International Conference on Interdisciplinary Science for Innovative Air Traffic Management. Florida: http://hal-enac.archives-ouvertes.fr/hal-00912772/PDF/ Chaimatanan/ISIATM2012.pdf, 2012.

[20] — , "Strategic deconfliction of aircraft trajectories," in ISIATM 2013, the 2nd International Conference on Interdisciplinary Science for Innovative Air Traffic Management. Toulouse (France): http://hal-enac. archives-ouvertes.fr/hal-00868450/PDF/isiatm2013/submission/123.pdf, 2013.

[21] _ - "A hybrid metaheuristic optimization algorithm for strategic planning of $4 \mathrm{~d}$ aircraft trajectories at the continental scale," Computational Intelligence Magazine, IEEE, vol. 9, no. 4, pp. 46-61, Nov 2014.

[22] J. K. Kuchar and L. C. Yang, "A review of conflict detection and resolution modeling methods," IEEE Transactions on Intelligent Transportation Systems, vol. 1, no. 4, pp. 179-189, 2000.

[23] S. Chaimatanan, Planification Stratégique de Trajectoires D'avions, 2014. [Online]. Available: https://books.google.co.th/books?id=efgcrgEACAAJ 